\title{
VALOR PROGNÓSTICO DO LINFONODO METASTÁTICO E SUA RELAÇÃO COM A SOBREVIDA LIVRE DE DOENÇA NO CÂNCER DE CABEÇA E PESCOÇO. ESTUDO DE 668 CASOS
}

\author{
PROGNOSTIC VALUE OF METASTATIC LYMPHNODE AND ITS RELATION WITH \\ FREE DISEASE SURVIVAL IN HEAD AND NECK CANCER. STUDY OF 668 CASES
}

\author{
Marcos Ribeiro de Magalhães ${ }^{1}$ \\ Ali Amar ${ }^{1}$ \\ Josias de Andrade Sobrinho ${ }^{2}$ \\ Marcos Brasilino de Carvalho ${ }^{3}$ \\ Abrão Rapoport-TCBC-SP ${ }^{4}$ \\ Odilon Victor Porto Denardin ${ }^{5}$
}

\begin{abstract}
RESUMO: Objetivo: Estudo retrospectivo e comparativo da sobrevida livre de doença, de 31 pacientes com metástase cervical de lesão primária oculta, submetidos a esvaziamento cervical e 637 pacientes submetidos ao mesmo procedimento, para o tratamento de câncer de cavidade oral, orofaringe e hipofaringe, operados no Serviço de Cirurgia de Cabeça e Pescoço do Complexo Hospitalar Heliópolis, São Paulo, Brasil, entre 1977 e 1995. Método: Levando-se em consideração o fator N do TNM (UICC-AJC 1997) para os pacientes com lesões primárias ocultas, e os fatores $\mathrm{T}$ e $\mathrm{N}$ para os com lesões primárias identificadas, foram confeccionadas as curvas de sobrevida livre de doença pelo método de Kaplan \& Meier e comparadas pelo teste de log-rank. Resultados: Comparamos a sobrevida livre de doença (SLD) das lesões de boca (b), orofaringe (o) e hipofaringe (h) com primário oculto (po) através de nove curvas considerando valores de p; variando os parâmetros Conclusões: verificadas as diferentes curvas de sobrevida livre, aferiu-se que não houve diferenças significantes na sobrevida livre de doença nas nove curvas planejadas (todos valores de $\mathrm{p}>0,05$ ), nos pacientes submetidos a esvaziamento cervical por primário oculto ou lesão identificada na boca, orofaringe e hipofaringe.
\end{abstract}

Descritores: Sobrevida livre de doença; Metástase; Cabeça e pescoço.

\section{INTRODUÇÃO}

A ocorrência de metástase cervical com lesão primária desconhecida é incomum, e tem incidência semelhante à encontrada quando se considera toda economia humana, situando-se em torno de $5 \%$ em nosso meio ${ }^{1}$, o que é referendado por grandes séries da literatura ${ }^{2}$ que relataram incidência de $3 \%$ a $9 \%{ }^{3}$. Tem, no entanto, peculiaridades, como o predomínio de carcinomas epidermóides em contraste com o dos adenocarcinomas quando se consideram todos os sítios corpóreos ${ }^{3}$. Outra diferença marcante é o fato de a metástase cervical com primário oculto ser uma entidade que permite a instituição de tratamentos curativos, encerrando surpreendentes taxas de sobrevida, sobretudo se considerarmos que a presença de metástase cervical determina a inclusão desses pacientes entre os "casos avançados" (estádio clínico III ou IV) de cabeça e pescoço ${ }^{4}$.

A fisiopatologia da metastatização na ausência de lesão primária identificável permanece desconhecida, afirmando-se que tais metástases ou ocorreram tão precocemente que não houve tempo hábil para manifestação clínica da doença local, ou a lesão primária apresentou regressão,

1. Mestre em Cirurgia de Cabeça e Pescoço, Hospital Heliópolis, Hosphel, São Paulo.

2. Cirurgião do Serviço de Cirurgia de Cabeça e Pescoço, Hospital Heliópolis, São Paulo.

3. Chefe do Serviço de Cirurgia de Cabeça e Pescoço, Hospital Heliópolis, Hosphel, São Paulo.

4. Docente-Livre em Cirurgia de Cabeça e Pescoço, Hospital Heliópolis, São Paulo.

5. Doutor em Endocrinologia, Hospital Heliópolis, São Paulo.

Recebido em 06/12/2000

Aceito para publicação em 27/03/2001

Trabalho realizado no Serviço de Cirurgia de Cabeça e Pescoço do Complexo Hospitalar Heliópolis, Hosphel, São Paulo, SP. 
análoga àquela demonstrada em melanomas malignos, ou, ainda, a manifestação primária é a própria lesão cervical ${ }^{5}$.

O objetivo deste trabalho é comparar a sobrevida livre de doença entre os pacientes submetidos a esvaziamento cervical por metástase cervical com lesão primária desconhecida, com aqueles nos quais a lesão inicial situava-se nas vias aerodigestivas superiores.

\section{MÉTODO}

Foram analisados 668 pacientes de um total de 1.043 submetidos a esvaziamento cervical por carcinoma espinocelular e indiferenciado, no Serviço de Cirurgia de $\mathrm{Ca}$ beça e Pescoço do Complexo Hospitalar Heliópolis, entre 1977 e 1995, reestadiados pelo sistema TNM UICC/AJC (1997) assim distribuídos:

- 31 com lesão primária desconhecida (Tx N1-3 M0).

- 347 com neoplasias de boca (T1-4 N0-3 M0).

- 123 com neoplasias de orofaringe (T1-4 N0-3 M0).

- 167 com neoplasias da hipofaringe (T1-4 N0-3 M0).

Os pacientes com linfonodopatia cervical, supeita de ser metastática, foram submetidos a exame das vias aerodigestivas superiores por, pelo menos, dois assistentes. $\mathrm{Na}$ ausência de lesão identificável, foram submetidos à fibroscopia e estudos radiográficos e/ou tomográficos. Persistindo desconhecida a lesão primária, foram submetidos à biopsia incisional ou excisional. Naqueles em que se identificou a lesão primária em boca, orofaringe e hipofaringe, a despeito da queixa ser local, ou cervical, realizou-se biopsia da lesão, ambulatorialmente, quando factível, ou em ambiente hospitalar, com sedação ou anestesia geral.

Os pacientes com metástase cervical com lesão primária desconhecida foram submetidos a esvaziamento cervical das cadeias I, II, III, IV e V, enquanto que os que apresentavam lesão identificável em boca, orofaringe ou hipofaringe à ressecção da lesão primária e a esvaziamento cervical, em monobloco ou dibloco. Nos casos $\mathrm{N}^{+}, \mathrm{o}$ esvaziamento contemplou as cadeias I, II, III, IV e V, e nos casos $\mathrm{N}^{-}$algumas cadeias foram poupadas, realizando-se esvaziamentos parciais.

No pós-operatório, os pacientes com pescoço positivo, aqueles com margens cirúrgicas comprometidas e com lesão extensa, receberam indicação de radioterapia.

O diagnóstico pré-operatório firmou-se pela realização de biopsias cujas lâminas foram coradas por hematoxilina-eosina. Após a realização do tratamento cirúrgico, os linfonodos foram dissecados das peças cirúrgicas frescas, e analisados à microscopia óptica corados por hematoxilina e eosina, assim como as lesões primárias identificadas.

Construíram-se nove curvas de sobrevida livre de doença, pelo método de Kaplan-Mayer, dos pacientes submetidos a esvaziamento cervical sem constatação de lesão primária, e dos pacientes submetidos a esvaziamento cervical por lesão identificada em cavidade oral, orofaringe e hipofaringe, levando-se em consideração os parâmetros T e N (tamanho do tumor e extensão de envolvi- mento linfonodal, respectivamente) da classificação TNM da UICC/AJCC de 1997 como se segue:

- Curva 1: pacientes Tx N1-3 versus T1-4 N0-3

- Curva 2: pacientes Tx N1-3 versus T1-4 N1-3

- Curva 3: pacientes Tx N1-3 versus T1-2 N0-3

- Curva 4: pacientes Tx N1-3 versus T1-2 N1-3

- Curva 5: pacientes Tx N1-3 versus T3-4 N0-3

- Curva 6: pacientes Tx N1-3 versus T3-4 N1-3

- Curva 7: pacientes Tx N1-2a versus T1-4 N0,1,2a

- Curva 8: pacientes Tx N1-2a versus T1-4 N0

- Curva 9: pacientes Tx N1-2 a versus T1-2 N0

As curvas foram comparadas pelo teste de log-rank, considerando-se significativo $\mathrm{p}<0,05$.

\section{RESULTADOS}

Todos os pacientes foram submetidos a esvaziamento cervical, sendo assim divididos:

- 31 pacientes com lesão primária oculta, sendo 29 homens e duas mulheres, idade média de 54,84 anos (intervalo de confiança de $95 \%=51,35-58,33$ anos).

- 637 pacientes com lesão primária diagnosticada:

- 2a. 34 na cavidade oral, sendo 30 homens e quatro mulheres; idade média de 54,96 anos (I C 95\% = 53,89$56,03)$.

- 2b: 123 no orofaringe, sendo 112 homens e 11 mulheres, idade média de 55,31 anos (IC 95\% = 53,65-56,96).

- 2c: 167 no hipofaringe, sendo 157 homens e dez mulheres, idade média de 55,93 anos (IC 95\% = 54,49-57,37).

Todos os 668 pacientes com carcinoma epidermóide e indiferenciado foram submetidos a esvaziamento cervical, estando assim distribuídos pela classificação TNM da UICC/AJC de 1997, sendo considerados os parâmetros N e pN para lesões primária oculta, boca, orofaringe e hipofaringe (Tabelas 1 e 2) e parâmetro T para as lesões primária oculta, e boca, orofaringe e hipofaringe (Tabelas 3 e 4 ).

Quanto à evolução no seguimento após cinco anos, tivemos:

1. Grupo com Lesão Primária Oculta (31 casos): nove vivos assintomáticos, 15 vivos com doença, quatro mortos com câncer, dois mortos por intercorrência, um vivo com segunda lesão.

2. Grupo com Lesão Primária Diagnosticada

2.1 Cavidade Oral (347 casos): 159 vivos assintomáticos, 84 vivos com câncer, 62 mortos com câncer, 32 mortos por intercorrência, dez mortos por segunda lesão.

2.2 Orofaringe (123 casos): 50 vivos assintomáticos, 29 vivos com câncer, 22 mortos com câncer, 20 mortos por intercorrência, dois vivos com segunda lesão.

2.3 Hipofaringe (167 casos): 62 vivos assintomáticos, 49 vivos com câncer, 25 mortos com câncer, 25 mortos por intercorrência, dois vivos com segunda lesão. 
$\mathrm{Na}$ avaliação dos resultados comparativos entre os grupos com primária conhecida ou não diagnosticada, a construção de nove curvas, para a so- brevida livre de doença (SLD) demonstrou ausência de significância estatística $(\mathrm{p}<0,05)$ através dos Gráficos 1 a 9 .

Tabela 1

Distribuição dos pacientes submetidos a esvaziamento cervical por sítio da lesão primária e fator $\mathrm{N}$

\begin{tabular}{l|r|r|r|r|r|r|r|r}
\hline & $N 0$ & $N 1$ & $N 2 a$ & $N 2 b$ & $N 2 c$ & N3 & NX & Total \\
\hline P. oculto & & 4 & 7 & 0 & 2 & 16 & 0 \\
Cav. oral & 163 & 95 & 13 & 42 & 18 & 14 & 2 & 347 \\
Orofaringe & 38 & 29 & 10 & 7 & 20 & 15 & 4 & 123 \\
Hipofaringe & 35 & 41 & 25 & 20 & 14 & 30 & 2 & 167 \\
\hline Total & 236 & 169 & 55 & 71 & 54 & 75 & 8 \\
\hline
\end{tabular}

Tabela 2

Distribuição dos pacientes submetidos a esvaziamento cervical por sítio da lesão primária e fator pN

\begin{tabular}{l|r|r|r|r|r|r|r|r}
\hline & $p N 0$ & $p N 1$ & $p N 2 a$ & $p n 2 b$ & $p N 2 c$ & PN3 & $p N X$ & Total \\
\hline P. oculto & 0 & 3 & 2 & 13 & 2 & 11 & 0 \\
Cav. oral & 153 & 53 & 9 & 110 & 17 & 5 & 0 & 31 \\
Orofaringe & 36 & 10 & 4 & 40 & 17 & 15 & 1 & 0 \\
Hipofaringe & 29 & 13 & 4 & 77 & 18 & 26 & 123 \\
\hline Total & 218 & 79 & 19 & 240 & 54 & 57 & 1 \\
\hline
\end{tabular}

Tabela 3

Distribuição dos pacientes submetidos a esvaziamento cervical por sítio da lesão primária e fator T

\begin{tabular}{l|r|r|r|r|r|r|r}
\hline & $T 0$ & $T 1$ & $T 2$ & $T 3$ & $T 4$ & $T X$ & Total \\
\hline P. oculto & 0 & 0 & 0 & 0 & 0 & 31 & 31 \\
Cav. oral & 3 & 12 & 102 & 116 & 109 & 5 & 347 \\
Orofaringe & 1 & 8 & 41 & 51 & 22 & 0 & 123 \\
Hipofaringe & 2 & 6 & 22 & 92 & 40 & 5 & 167 \\
\hline Total & 6 & 26 & 165 & 259 & 171 & 41 & 668 \\
\hline
\end{tabular}

Tabela 4

Distribuição dos pacientes submetidos a esvaziamento cervical por sítio da lesão primária e fator pT

\begin{tabular}{l|c|c|c|c|c|r|r}
\hline & $p T 0$ & $p T 1$ & $p T 2$ & $p T 3$ & $p T 4$ & $P T X$ & Total \\
\hline P. oculto & 0 & 0 & 0 & 0 & 0 & 31 & 31 \\
Cav. oral & 3 & 12 & 97 & 96 & 134 & 5 & 347 \\
Orofaringe & 1 & 4 & 35 & 45 & 35 & 3 & 123 \\
Hipofaringe & 2 & 4 & 20 & 81 & 57 & 3 & 167 \\
\hline Total & 6 & 20 & 152 & 222 & 226 & 42 & 668 \\
\hline
\end{tabular}


Nos Gráficos 1 a 9 verificamos que houve ausência de significado estatístico para SLD no estudo comparativo do tumor primário oculto $(\mathrm{Tx})$ e aque- les localizados na boca, orofaringe e hipofaringe, mesmo considerados diferentes valores para metástase $(\mathrm{N})$.

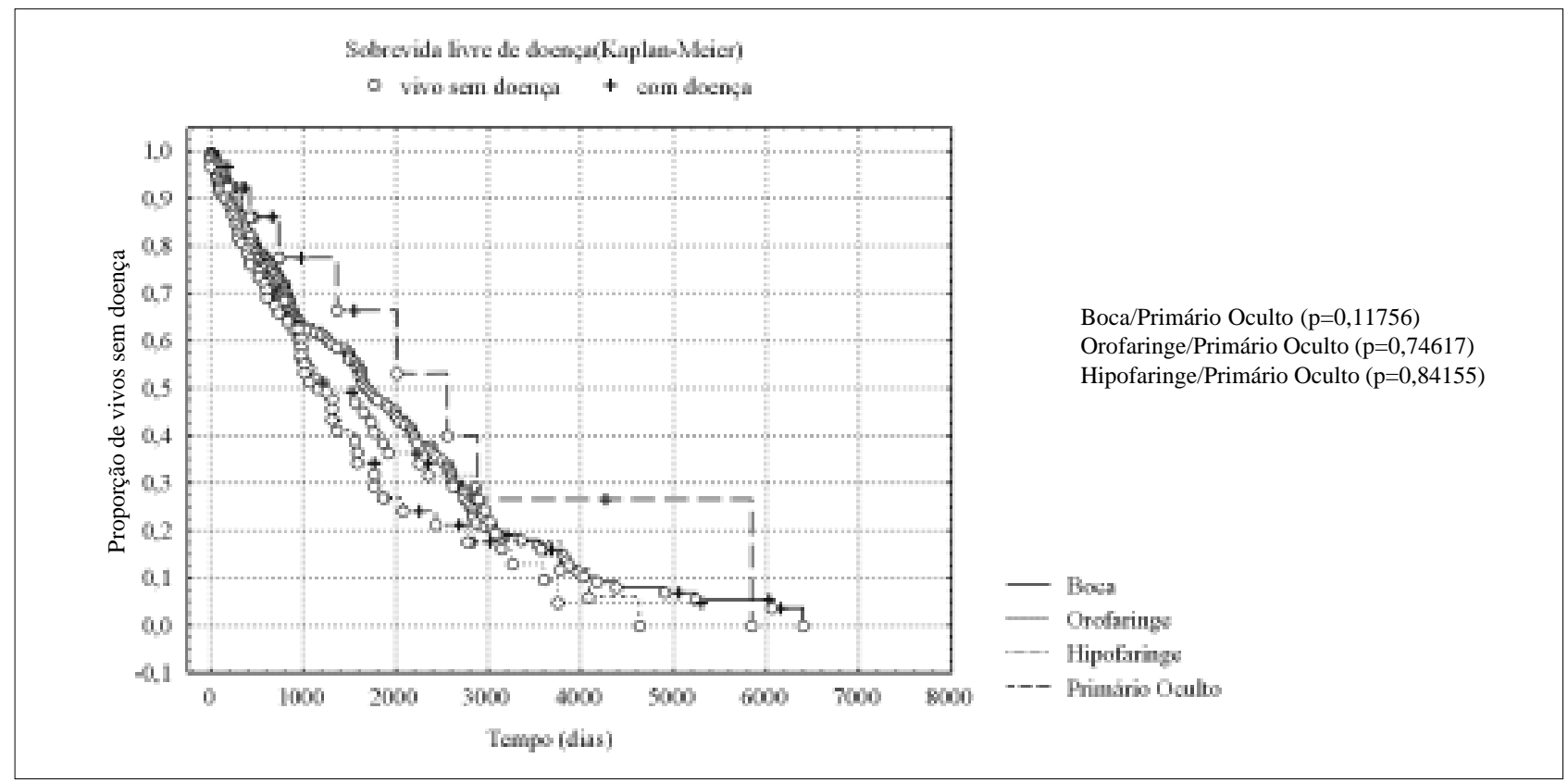

Gráfico 1 - Sobrevida livre de doença em pacientes com primário oculto (TX N1-3) e com lesão identificada em boca, orofaringe e hipofaringe (T1-4 NO-3), submetidos a esvaziamento cervical.

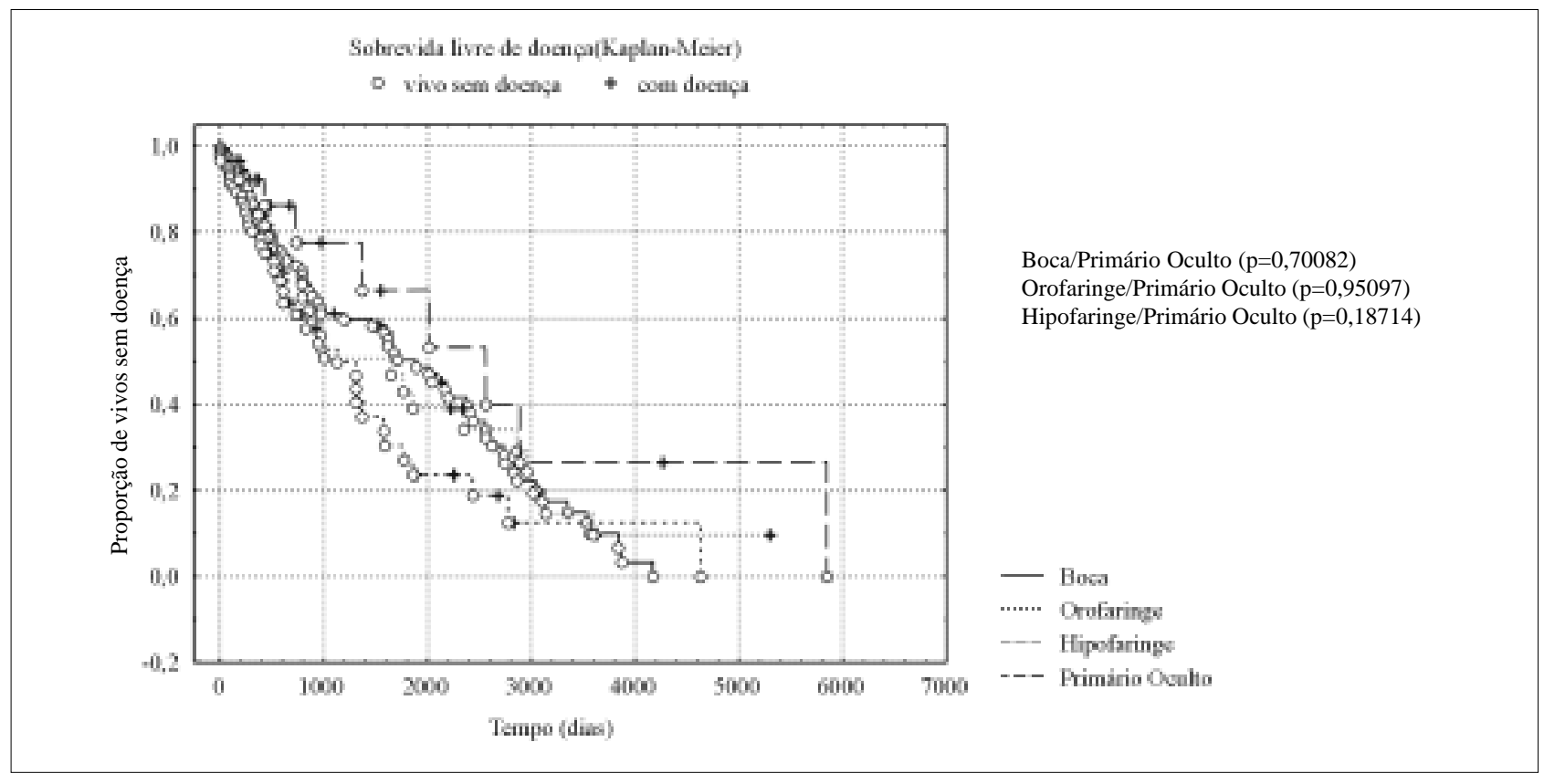

Gráfico 2 - Sobrevida livre de doença em pacientes com primário oculto (TX N1-3) e com lesão identificada em boca, orofaringe e hipofaringe (T1-4 N1-3), submetidos a esvaziamento cervical. 


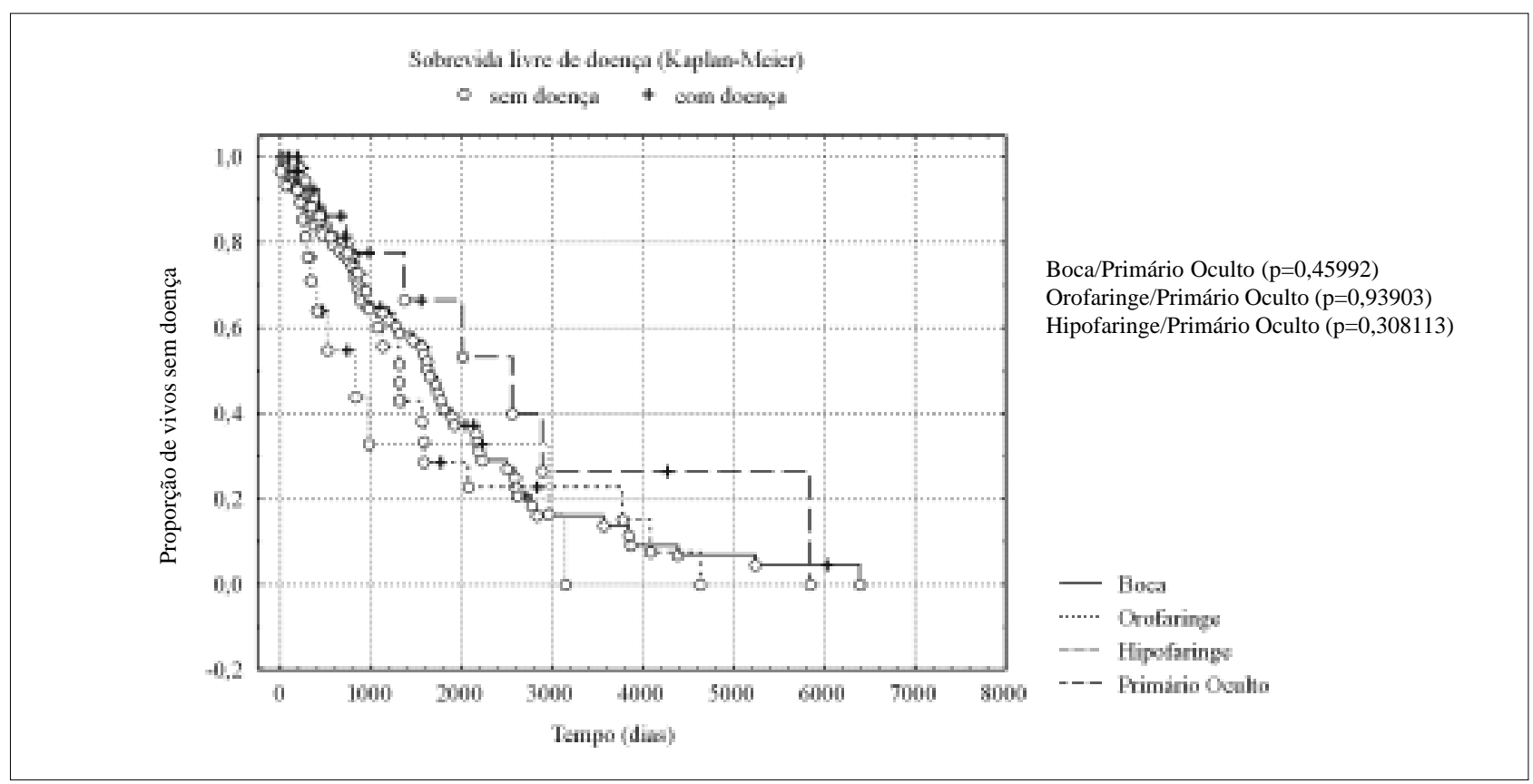

Gráfico 3 - Sobrevida livre de doença em pacientes com primário oculto (TX N1-3) e com lesão identificada em boca, orofaringe e hipofaringe (T1-2 N0-3), submetidos a esvaziamento cervical.

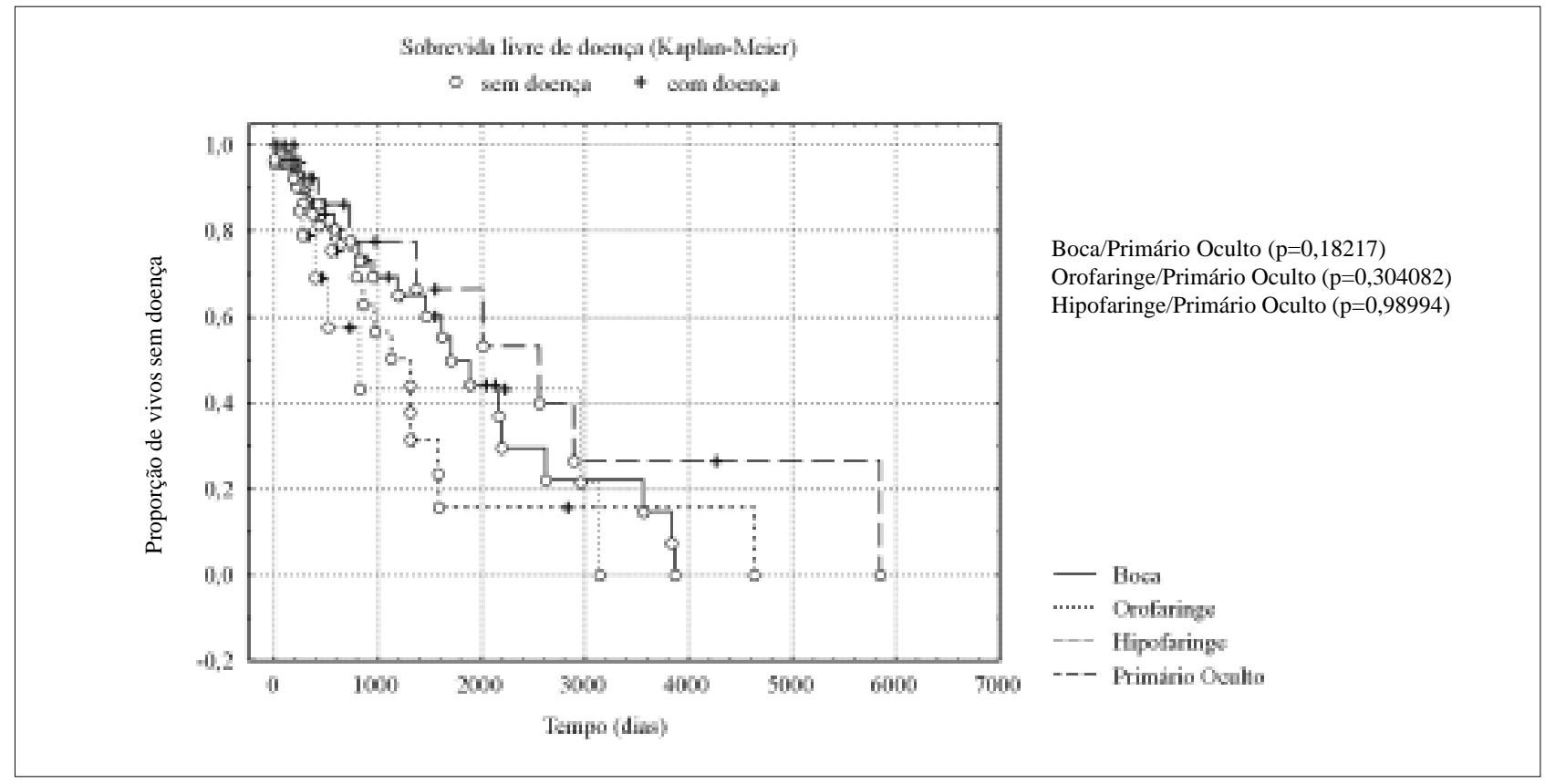

Gráfico 4 - Sobrevida livre de doença em pacientes com primário oculto (TX N1-3) e com lesão identificada em boca, orofaringe e hipofaringe (T1-2 N1-3), submetidos a esvaziamento cervical.

\section{DISCUSSÃO}

A constatação de metástase cervical na ausência de lesão primária identificada, está longe de ser raridade no cotidiano da cirurgia de cabeça e pescoço, ocorrendo de
$3 \%$ a $9 \%$ de metástase cervical em pacientes em que não se conseguiu, a despeito dos esforços propedêuticos, evidenciar lesão primária ${ }^{1}$. Da mesma forma, não é possível fazer um diagnóstico precoce, uma vez que a metástase cervical é determinante da inclusão desses pacientes nos estádios 


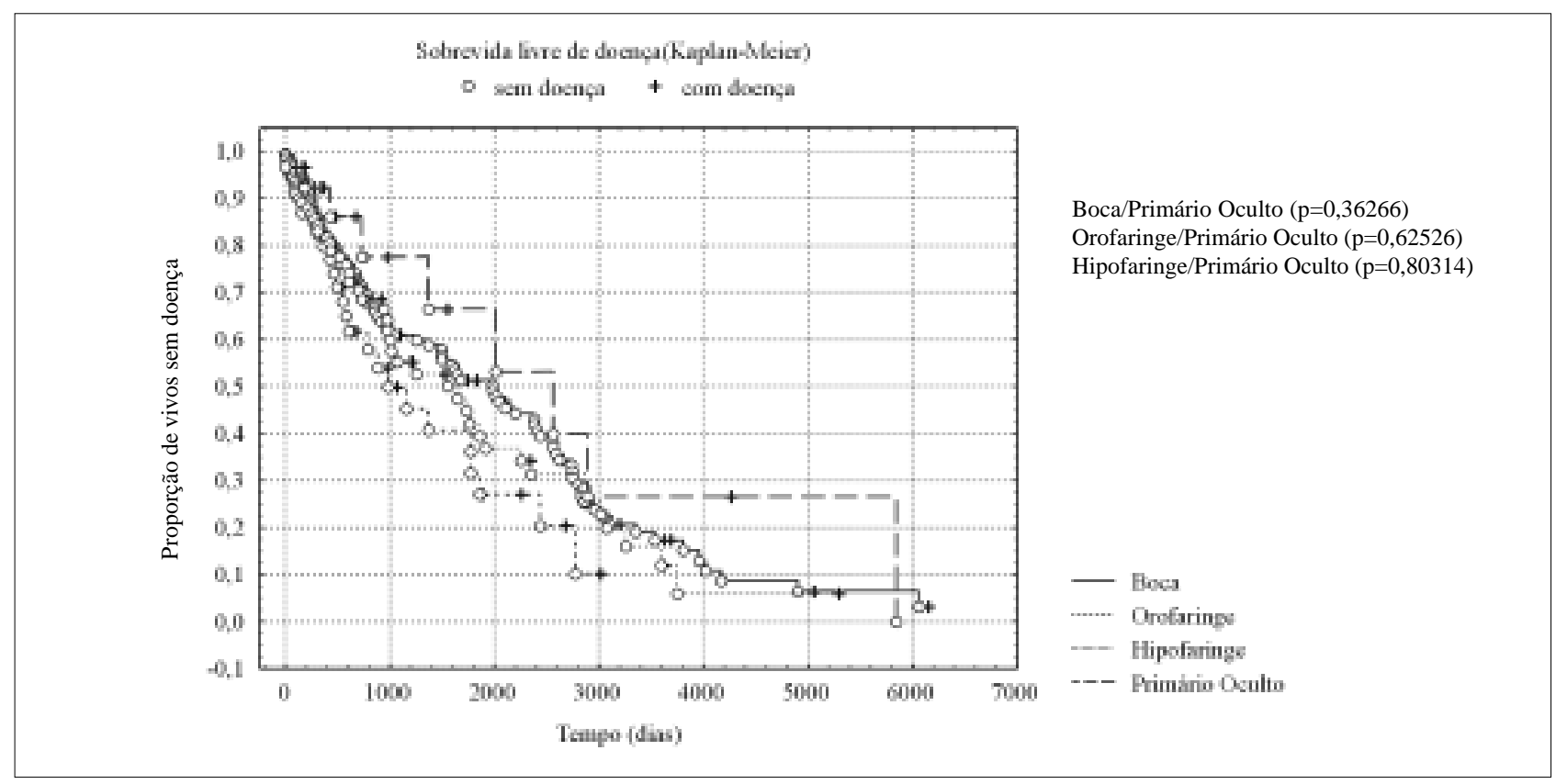

Gráfico 5 - Sobrevida livre de doença em pacientes com primário oculto (TX N1-3) e com lesão identificada em boca, orofaringe e hipofaringe (T3-4 NO-3), submetidos a esvaziamento cervical.

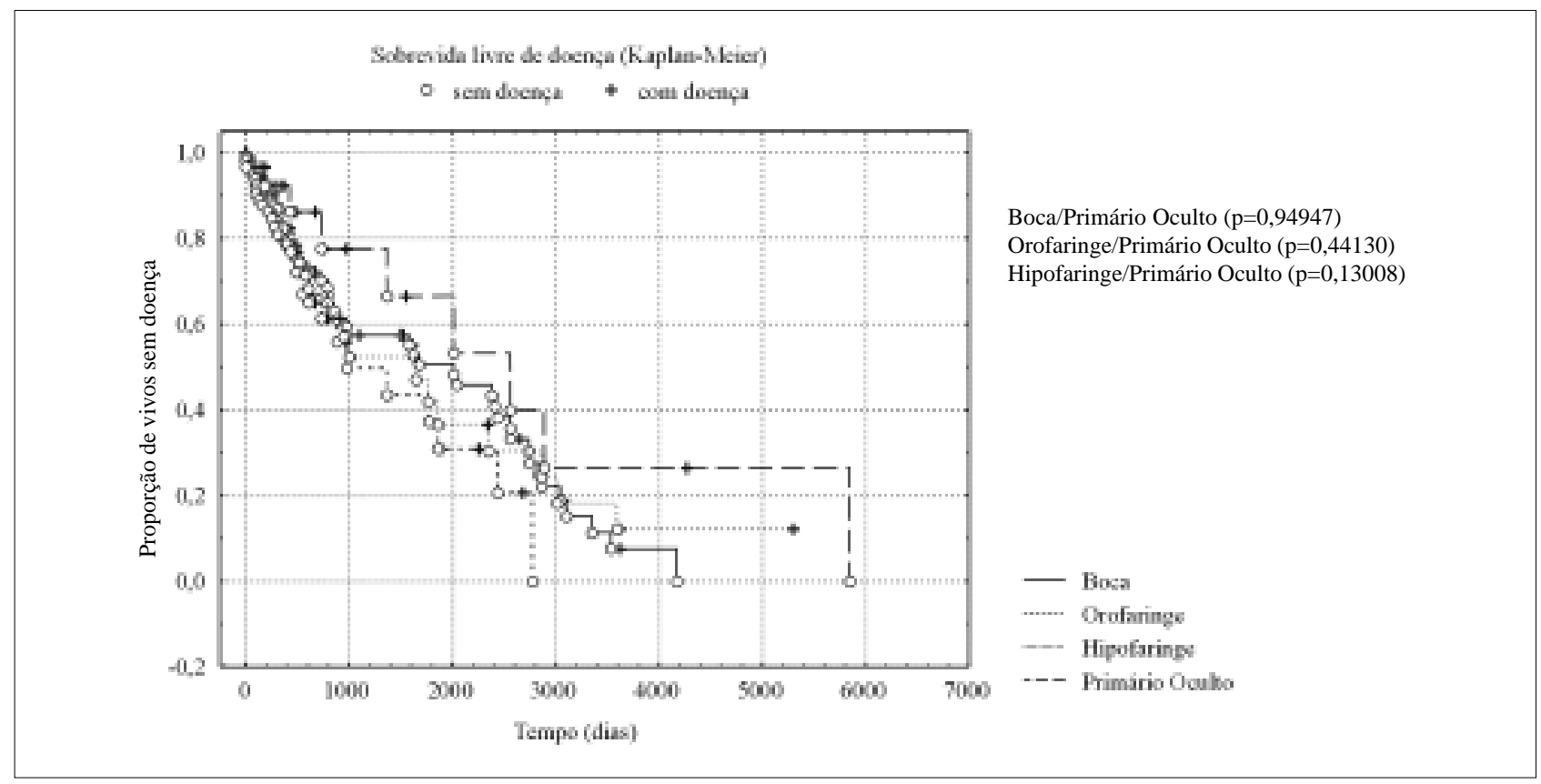

Gráfico 6 - Sobrevida livre de doença em pacientes com primário oculto (TX N1-3) e com lesão identificada em boca, orofaringe e hipofaringe (T3-4 N1-3), submetidos a esvaziamento cervical.

clínicos III ou IV, bem como não se dispõem de testes para rastreamento ${ }^{5}$.

Aceitar que, por sua capacidade de metastatizar na ausência de lesão primária identificável, essa entidade apresente maior agressividade, não encontrou respaldo em nossa casuística, em que não constatamos diferença estatisticamente significante entre as curvas de sobrevida livre de doença entre os pacientes submetidos a esvaziamento cervical por metástase cervical com lesão primária não reconhecida e aqueles submetidos ao mesmo procedimento, na presença de lesão primária diagnosticada. Não ignoramos o fato de que os pacientes selecio- 


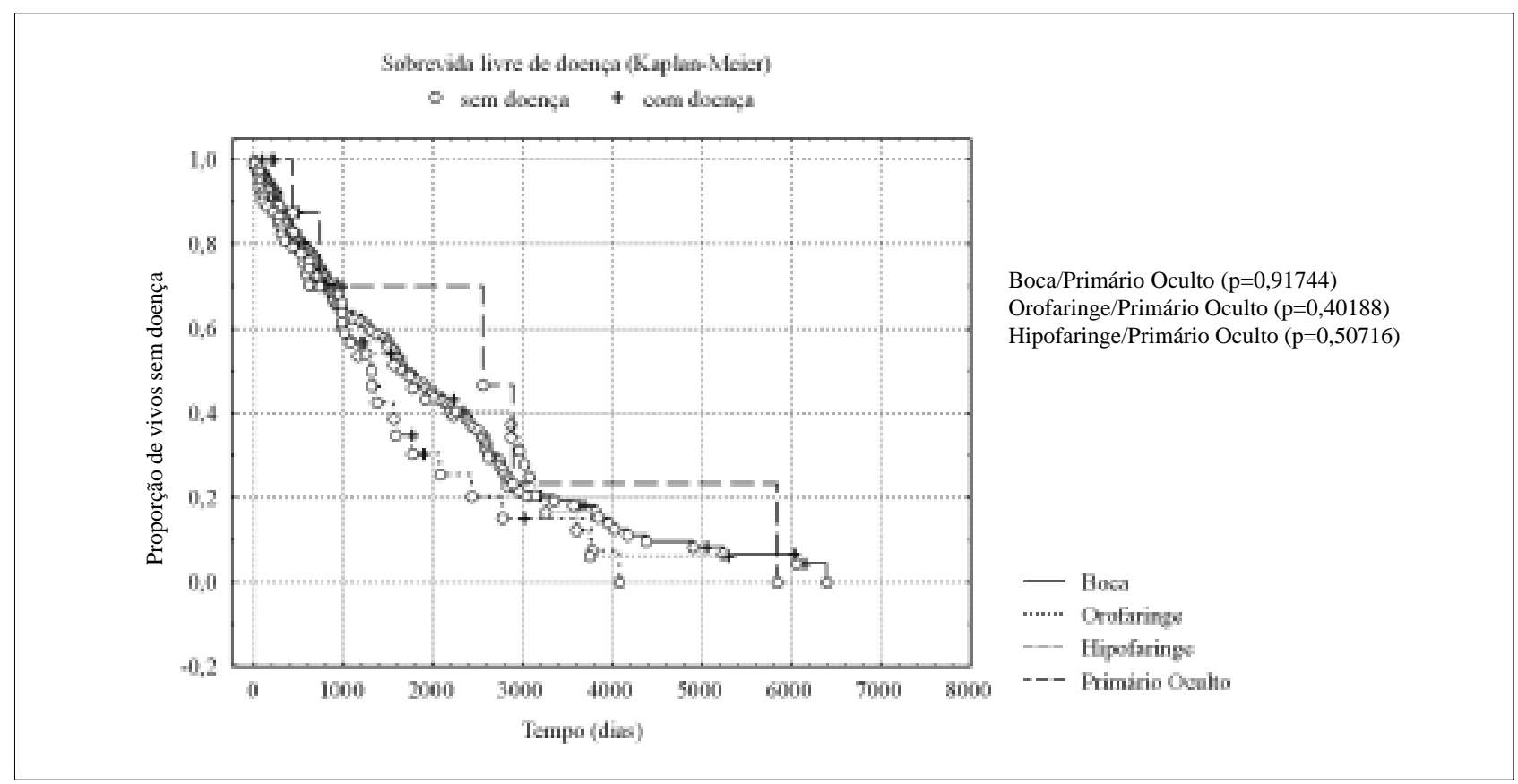

Gráfico 7 - Sobrevida livre de doença em pacientes com primário oculto (TX N1-2a) e com lesão identificada em boca, orofaringe e hipofaringe (T1-4 NO-2a), submetidos a esvaziamento cervical.

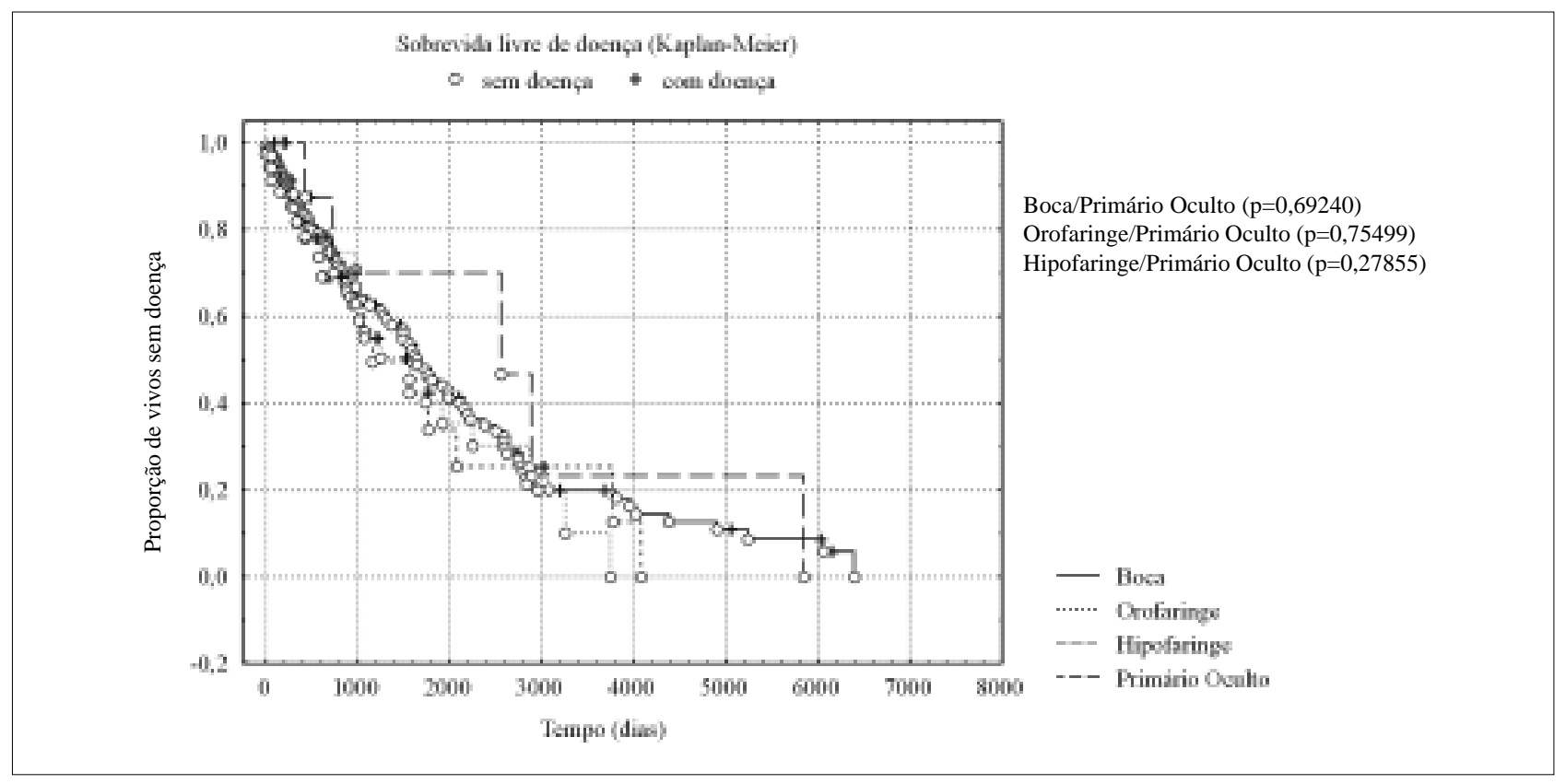

Gráfico 8 - Sobrevida livre de doença em pacientes com primário oculto (TX N1-2a) e com lesão identificada em boca, orofaringe e hipofaringe (T1-4 NO), submetidos a esvaziamento cervical.

nados para o tratamento cirúrgico, entre os que apresentavam metástase cervical com lesão primária não identificável, seguramente, apresentavam melhores condições clínicas e massas tumorais cervicais de menor extensão que a maioria dos pacientes que se apresentam para tratamento por essa entidade clínica ${ }^{6}$. No entanto, esse fato não deixa de ser aplicável aos pacientes com lesão primária conhecida, que, em nosso meio, freqüentemente se apresentam sem condições para o tratamento convencional. Dessa forma, aceitamos que aqueles pacientes selecionados para o tratamento cirúrgico inicial, com lesão primária conhecida ou não, sejam retratos de 


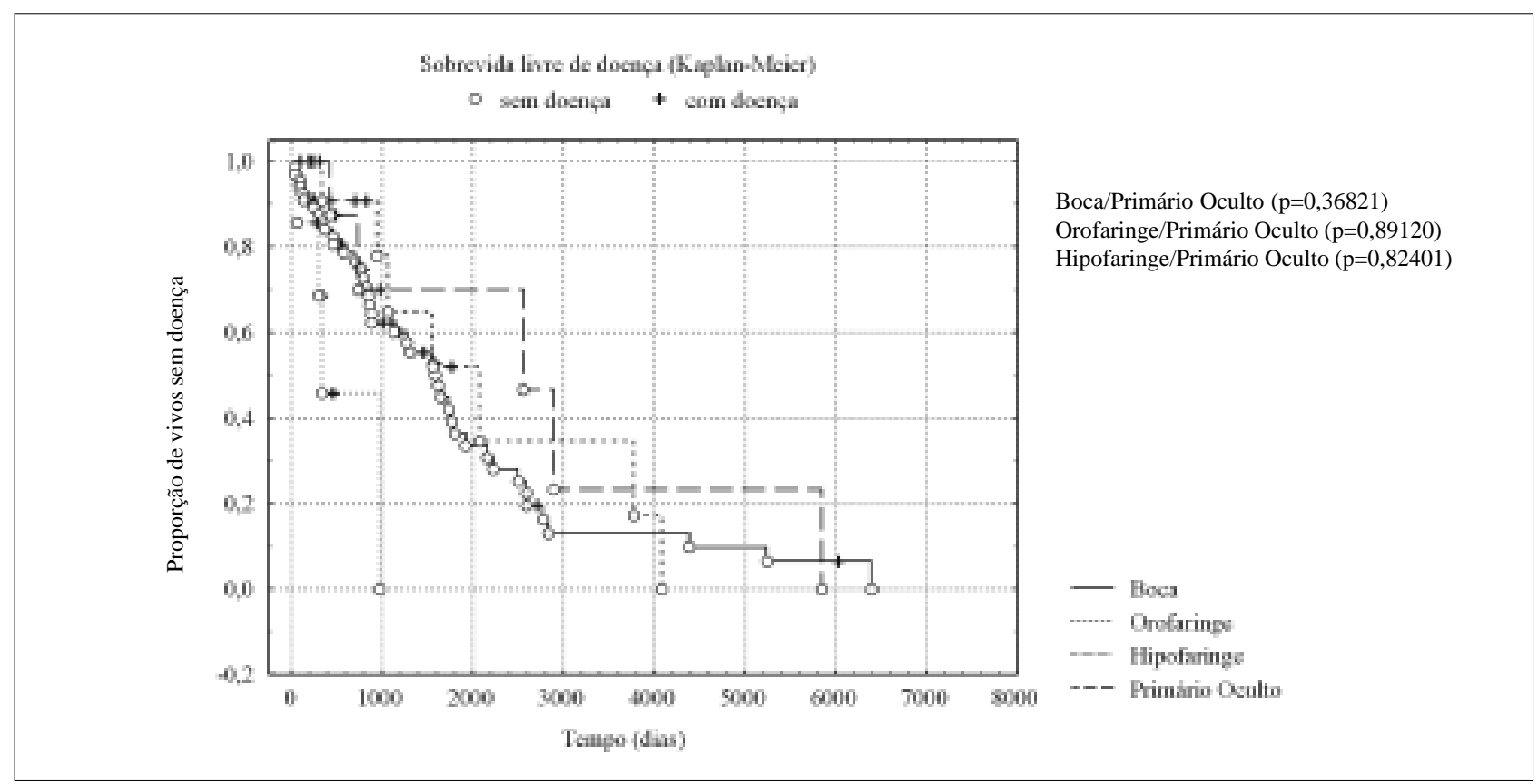

Gráfico 9 - Sobrevida livre de doença em pacientes com primário oculto (TX N1-2a) e com lesão identificada em boca, orofaringe e hipofaringe (T1-2 NO), submetidos a esvaziamento cervical.

uma fase da doença neoplásica, apresentando seguramente diferenças evolutivas, em especial as relacionadas ao indivíduo. Tal aquiescência não nos impede, entretanto, de verificarmos a sobrevida livre de doença semelhante entre os dois grupos, mesmos submetidos à estratificação quanto ao parâmetro $\mathrm{T}$ em relação àqueles com lesão primária conhecida. Concordante com a literatura, o comprometimento cervical, independente do tamanho tumoral, é determinante da evolução dos pacientes com câncer de cabeça e pescoço, o que se mostrou também aplicável aos pacientes com metástase cervical com lesão primária não identificável.

Merece destaque, em nossos resultados, o fato de a curva de sobrevida livre de doença entre pacientes com um único linfonodo cervical e com lesão primária oculta, não apresentar diferença estatisticamente significativa em relação às lesões identificadas em boca, orofaringe e hipofaringe, mesmo iniciais e com pescoço negativo. Tal fato leva-nos a inferir que a lesão primária oculta pode comportar-se como lesão primária, longe, no entanto, de acatar a hipótese de primária cervical. $\mathrm{O}$ pequeno número de pacientes no grupo de primário oculto, especialmente quando estratificado, representa um viés importante, tanto na discussão anterior, como em todo o trabalho.

A perda de seguimento é outro viés importante nos trabalhos que envolvem sobrevida, em especial no nosso meio, não sendo diferente neste trabalho. Tal problema foi contornado pela utilização de sobrevida livre de doença, considerando o estado do paciente no último retorno, o que determinou o não aparecimento da perda de seguimento.
A razão pela qual lesões, ou tão pequenas que não sejam identificáveis, ou com a capacidade de regressão, sejam capazes de permitir o processo da metastatização talvez deva ser buscada na imunologia e/ou biologia molecular, em especial no tocante à adesividade celular. A adesividade precária no local de origem explicaria uma precoce metastização. O fato de a ocorrência de metástase a distância entre os pacientes com lesão primária desconhecida, cuja histologia seja carcinoma espinocelular e não adenocarcinoma, ser semelhante aos com lesão identificável, sugere haver uma facilitação para o desenvolvimento dessas metástases nos linfonodos, talvez também relacionados à imunologia e/ou biologia molecular.

Muito embora desafie nossa compreensão, à luz dos conhecimentos da oncologia, a metástase cervical com lesão primária desconhecida, oculta, não-identificável, como se queira denominar, não representa ameaça maior ao paciente que, por capacitação genotípica e fatores ambientais, foi capaz de desenvolver um carcinoma de vias aerodigestivas superiores. Não é despropositado imaginar que essa entidade clínica pode carregar em si respostas para muitas dúvidas da carcinogênese e metastatização, bem como abrir portas terapêuticas.

Em síntese, o estudo das curvas de sobrevida livre de doença, entre pacientes submetidos a esvaziamento cervical por metástase cervical, na ausência de lesão identificável, comparados com pacientes submetidos a esvaziamento cervical por lesão identificada em boca, orofaringe ou hipofaringe, na presença ou não de metástase cervical clinicamente evidente, não mostrou diferenças estatisticamente significativas. 


\begin{abstract}
Background: Comparative analysis of a retrospective study of the free disease survival of 31 patients with cervical metastasis with unknown primary tumor, and 637 patients with head and neck cancer of the mouth, oropharynx and hypopharynx submitted to neck dissection, in the Head and Neck Service of the Heliópolis Hospital Complex, São Paulo, Brazil, between 1977 and 1995. Method: Free disease survival curves were determined by the KAPLAN \& MEIER method, taking into consideration the $N$ factor of TNM classification (UICC - AJC 1997) for the patients with unknown tumor sites, and the T and $N$ factors for those diagnosed with primary tumor. These curves were compared by the log-rank test. Results: the comparison of free disease survival (FDS) of patients with cancer of the mouth $(m)$, oropharynx $(o)$ and hypopharynx with unknown primary (up) lesion were established through 9 curves, considering values of p, with T and $N$ parameters. Conclusions: the verifications of differents survival curves did not detect statistical diferences for the Free Disease Survival in 9 planned curves (all values of p more than 0,05), for patients with cervical metastasis with unknown primary tumor, and those with mouth, oropharynx and hypopharynx tumors, submitted to neck dissection.
\end{abstract}

Key Words: Free disease survival; Metastasis; Head and neck.

\title{
REFERÊNCIAS
}

1. Amar A, Magalhães MR, Rapoport A et al. Metástase cervical de tumor primário oculto: Estudo de 107 casos. Rev Col Bras Cir., 2000; 27:90-96.

2. De Braud F, Al-Sarraf M. Diagnosis and management of squamous cell carcinoma of unknown primary tumor site of the neck. Semin Oncol, 1993; 20: 273-278.

3. Lefebvre J, Coche-Dequeant B, Van JT et al. Cervical lymphonodes from an unknown primary tumor in 190 patients. Am J Surg, 1990; 160: 443-446.

4. Hainsworth JD, Greco FA. Introduction: Unknown primary tumor. Semin Oncol, 1993; 20:205.

5. Davidson BJ, Harter W, O'Malley BB. In: Harrison LB, Sessions RB, Hong WK. Head and neck cancer: a multidisciplinary approach. Lippincott-Raven Publishers - Philadelphia - , 1999; 391-410.
6. Bataini JP, Rodriguez J, Jaulerry C et al. Treatment of metastatic neck nodes secondary to an occult epidermoid carcinoma of the head and neck. Laryngoscope, 1987; 97: 1080-1084.

Endereço para correspondência:

Prof. Dr. Abrão Rapoport

Praça Amadeu Amaral, 47 - cj. 82 - Paraíso

01327-010 - São Paulo-SP

E-mail: cpgcp.hosphel@attglobal.net 